\title{
Heat flow, weighted Bergman spaces, and real analytic Lipschitz approximation
}

\author{
By Wolfram Bauer at Hannover and Lewis A. Coburn at Buffalo
}

\begin{abstract}
We show that, for $f$ any uniformly continuous (UC) complex-valued function on real Euclidean $n$-space $\mathbb{R}^{n}$, the heat flow $\tilde{f}^{(t)}$ is Lipschitz for all $t>0$ and $\tilde{f}^{(t)}$ converges uniformly to $f$ as $t \rightarrow 0$. Analogously, let $\Omega$ be any irreducible bounded symmetric (Cartan) domain in complex $n$-space $\mathbb{C}^{n}$ and consider the Bergman metric $\beta(\cdot, \cdot)$ on $\Omega$. For $f$ any $\beta$-uniformly continuous function on $\Omega$, we show that there is a Berezin-Harish-Chandra flow of real analytic functions $B_{\lambda} f$ which is $\beta$-Lipschitz for each $\lambda \geq p$ ( $p$, the genus of $\Omega$ ) and $B_{\lambda} f$ converges uniformly to $f$ as $\lambda \rightarrow \infty$. For a certain subspace of UC we obtain stronger approximation results and we study the asymptotic behaviour of the Lipschitz constants.
\end{abstract}

\section{Introduction}

The problem of Lipschitz approximation to uniformly continuous complex-valued functions on metric spaces $X$ is an old one, going back - at least - to Lebesgue. It is known [5] that, for "metrically convex" $X$ (including complete Riemannian manifolds), Lipschitz functions are uniformly dense in the uniformly continuous functions. The standard density construction guarantees no particular smoothness of the Lipschitz approximants, instead resembling piecewise linear approximation on the reals.

In this paper, we consider the subspaces $\Omega$ of complex $n$-space $\mathbb{C}^{n}$, where $\Omega=\mathbb{C}^{n}$ or $\Omega$ is an irreducible bounded symmetric domain (BSD) in $\mathbb{C}^{n}$ with Bergman metric $\beta(\cdot, \cdot)$. We obtain real-analytic Lipschitz approximants to arbitrary $\beta$-uniformly continuous functions $f$ by using the heat-flow for $\mathbb{C}^{n}$ and the Berezin-Harish-Chandra flow when $\Omega$ is an arbitrary irreducible BSD. For the heat flow $\tilde{f}^{(t)}$, it is reasonable to expect that $\tilde{f}^{(t)} \rightarrow f$ uniformly as $t \rightarrow 0$. It is less clear that the $\tilde{f}^{(t)}$ are Lipschitz. The Berezin-Harish-Chandra flows for $f$ $\beta$-uniformly continuous on BSD $\Omega$, discussed in Section 4, are natural analogs of the heat flow.

Bounded symmetric domains (BSDs) are Hermitian symmetric spaces of non-compact type $[4,7,9,10]$. There is a standard classification of BSDs going back to H. Cartan. We work in the Harish-Chandra realization of BSDs as bounded convex domains $\Omega$ containing the origin 0

The first named author has been supported by an "Emmy-Noether scholarship" of the DFG (Deutsche Forschungsgemeinschaft). 
of $\mathbb{C}^{n}$ and invariant under the map $z \rightarrow \lambda z$ for $\lambda$ in $\mathbb{C}$ and $|\lambda|=1$. The group $\operatorname{Aut}(\Omega)$ of biholomorphic automorphisms of $\Omega$ is transitive. In particular, for each $w$ in $\Omega$, there is an automorphism $\varphi_{w}$ so that $\varphi_{w} \circ \varphi_{w}=$ identity and $\varphi_{w}(0)=w$.

Here, we consider BSDs $\Omega$ with normalized Lebesgue measure $d v(z)$, in $\mathbb{C}^{n}$, or $\Omega=\mathbb{C}^{n}$ with normalized Gaussian measure $d \mu(z)=\exp \left[-|z|^{2}\right] \pi^{-n} d v(z)$. It is well known that, for any bounded domain $\Omega$ in $\mathbb{C}^{n}$, the space of complex analytic functions in $L^{2}(\Omega, d v)$, denoted $L_{\text {an }}^{2}(\Omega, d v)$, is a Bergman space: for every $w$ in $\Omega$ there is a function $K(\cdot, w)$ in $L_{\text {an }}^{2}(\Omega, d v)$ so that, for all $f$ in $L_{\text {an }}^{2}(\Omega, d v)(\bar{a}$ is the complex conjugate of $a)$,

$$
f(w)=\int_{\Omega} f(z) \overline{K(z, w)} d v(z) \equiv\langle f, K(\cdot, w)\rangle .
$$

The corresponding formula holds for $\left(\mathbb{C}^{n}, d \mu\right)$ and $f$ entire in $L^{2}\left(\mathbb{C}^{n}, d \mu\right)$ where we say $f$ is in $H^{2}\left(\mathbb{C}^{n}, d \mu\right)$. It is standard that $K(w, z)=\overline{K(z, w)}$. For $\Omega=\mathbb{D}$, the open unit disc in $\mathbb{C}$ with normalized Lebesgue measure, we have

$$
K(z, w)=(1-z \bar{w})^{-2} .
$$

For $\Omega=\mathbb{C}^{n}$ with normalized Gaussian measure $d \mu(z)$, we have

$$
K(z, w)=\exp (z \cdot w) \quad\left(z \cdot w=z_{1} \overline{w_{1}}+z_{2} \overline{w_{2}}+\cdots+z_{n} \overline{w_{n}}\right) .
$$

For $\Omega$ a BSD in $\mathbb{C}^{n}$ with normalized Lebesgue measure $d v$, or $\Omega=\mathbb{C}^{n}$ with normalized Gaussian measure $d \mu, K(z, 0)=1$ and $K(z, z) \geq 1$ for all $z$ in $\Omega$. Moreover, we have $\lim _{z \rightarrow \partial \Omega} K(z, z)=\infty$. The functions $K(z, z)$ determine a complete Riemannian metric on $\Omega$ by the formula

$$
g_{i j}(z)=\frac{\partial^{2}}{\partial z_{i} \partial \bar{z}_{j}} \log K(z, z), \quad z \in \Omega .
$$

In the case that $\Omega$ is the open unit ball, the Bergman metric is the usual hyperbolic metric, when $\Omega=\mathbb{C}^{n}$, the Bergman metric is the usual Euclidean metric. The Bergman metric induces a distance function $\beta(\cdot, \cdot)$ on $\Omega$. For BSD $\Omega$, [4, Theorem E], the function $\beta(0, \cdot)$ is in $L^{p}(\Omega, d v)$ for all $p>0$. The corresponding result holds for $\left(\mathbb{C}^{n}, d \mu\right)$ by direct calculation. For $\Omega=\mathbb{D}$, the open unit disc in $\mathbb{C}$, it is standard that

$$
\beta(0, z)=\frac{1}{\sqrt{2}} \log \left(\frac{1+|z|}{1-|z|}\right)
$$

while for $\Omega=\mathbb{C}^{n}, \beta(0, z)=|z|$ and $\varphi_{w}(z)=w-z$. For all BSDs $\Omega$ and for $\mathbb{C}^{n}$,

$$
\beta\left(\varphi_{w} z, \varphi_{w} y\right)=\beta(z, y)
$$

for all $z, y$ in $\Omega$ and each $w$ in $\Omega$. Note that, for $\partial \Omega$ the boundary of $\Omega$,

$$
\lim _{z \rightarrow \partial \Omega} \beta(0, z)=\infty .
$$

The $\beta$-metric topology on BSD $\Omega$ is equivalent to the usual Euclidean topology inherited from $\mathbb{C}^{n}$. In particular, the closed metric balls $E(0, R)=\{z \in \Omega: \beta(0, z) \leq R\}$ are compact. A function $f$ from $\Omega$ to $\mathbb{C}$ is Lipschitz (Lip) if there is a constant $D_{f}$ so that for all $z, w$ in $\Omega$,

$$
|f(z)-f(w)| \leq D_{f} \beta(z, w)
$$


The function $f$ is uniformly continuous (UC) if, for each $\varepsilon>0$, there is a $\delta=\delta(f, \varepsilon)>0$ so that, for any $z, w$ in $\Omega,|f(z)-f(w)|<\varepsilon$ whenever $\beta(z, w)<\delta$. A continuous function $f$ is of bounded oscillation (BO) if there is a $C>0$ so that, for all $z, w$ in $\Omega$,

$$
|f(z)-f(w)| \leq C+C \beta(z, w) .
$$

As usual, we denote the bounded functions in $\operatorname{UC}(\Omega)$ by $\operatorname{BUC}(\Omega)$. The relation

$$
\operatorname{Lip}(\Omega) \subset \operatorname{UC}(\Omega)
$$

is standard.

The functions

$$
k_{w}(\cdot)=K(w, w)^{-\frac{1}{2}} K(\cdot, w)
$$

are unit vectors in $L_{\text {an }}^{2}(\Omega, d v)$ and $H^{2}\left(\mathbb{C}^{n}, d \mu\right)$. For BSDs $\Omega$, the functions $\left\{k_{w}(\cdot)\right\}$ are bounded for each $w$ in $\Omega$. For $\left(\mathbb{C}^{n}, d \mu\right)$, the $k_{w}$ are linear exponentials. We define the Berezin transform of $f$ by

$$
\tilde{f}(w)=\int_{\Omega} f \circ \varphi_{w}(z) d v(z)=\int_{\Omega} f(z)\left|k_{w}(z)\right|^{2} d v(z)
$$

with the analogous definition for $\Omega=\mathbb{C}^{n}$. This definition makes sense for $f$ and $|f|^{2}$ when $f$ is in $L^{2}(\Omega, d v)$ for BSD $\Omega$ and for $f$ and $|f|^{2}$ on $\mathbb{C}^{n}$ for $f$ in

$$
\tau\left(\mathbb{C}^{n}\right)=\left\{f: f k_{w} \in L^{2}\left(\mathbb{C}^{n}, d \mu\right) \text { for all } w \in \mathbb{C}^{n}\right\} .
$$

Note that $\widetilde{\left.f\right|^{2}}(w) \geq|\tilde{f}(w)|^{2}$ by an easy application of the Cauchy-Schwarz inequality.

Following [4], we say that $f$ has bounded mean oscillation on $\operatorname{BSD} \Omega\left(f\right.$ is in $\left.\mathrm{BMO}^{2}(\Omega)\right)$ if the continuous function

$$
\left.\operatorname{MO}(f, w) \equiv\left|\widetilde{\left.f\right|^{2}}(w)-\right| \tilde{f}(w)\right|^{2}=\left\{|f(\cdot)-\tilde{f}(w)|^{2}\right\} \sim(w)
$$

is bounded on $\Omega$ and we define a semi-norm on $\operatorname{BMO}^{2}(\Omega)$ by

$$
\|f\|_{\mathrm{BMO}}:=\sup _{z \in \Omega} \operatorname{MO}(f, z)^{\frac{1}{2}} .
$$

Again, there is a completely analogous definition in [1] for $\Omega=\mathbb{C}^{n}$.

For a BSD $\Omega$, we say that $f$ has vanishing mean oscillation at the boundary $\partial \Omega$ ( $f$ is in $\left.\operatorname{VMO}^{2}(\Omega)\right)$ if $\lim _{w \rightarrow \partial \Omega} \operatorname{MO}(f, w)=0$. Analogously, for $\left(\mathbb{C}^{n}, d \mu\right)$, we say $f$ has vanishing mean oscillation at $\infty\left(f\right.$ is in $\left.\operatorname{VMO}^{2}\left(\mathbb{C}^{n}\right)\right)$ if $\lim _{|w| \rightarrow \infty} \operatorname{MO}(f, w)=0$. Note that, trivially, all bounded measurable functions are in $\operatorname{BMO}^{2}(\Omega)$.

It is known ([4, Theorem 13], [1, Lemma 3.5]) that for $\Omega$ any BSD or $\Omega=\mathbb{C}^{n}$, we have

$$
\mathrm{BO}(\Omega) \subset \mathrm{BMO}^{2}(\Omega) .
$$

It is also known that, for $f$ in $\mathrm{BMO}^{2}(\Omega), \tilde{f}$ is Lipschitz. In fact, there is a decomposition of spaces

$$
\mathrm{BMO}^{2}(\Omega)=\mathrm{BO}(\Omega)+\mathrm{F}(\Omega)
$$

for all BSDs $\Omega$, where

$$
\mathrm{F}(\Omega)=\left\{f \in L^{2}(\Omega, d v): \widetilde{|f|^{2}} \text { is bounded }\right\}
$$

and analogously for $\Omega=\mathbb{C}^{n}$. 
In Section 2 we show that $\operatorname{UC}(\Omega)$ is in $\operatorname{BO}(\Omega)$, and therefore in $\mathrm{BMO}^{2}(\Omega)$ for $\Omega$ any BSD or $\Omega=\mathbb{C}^{n}$. We consider the class of "generalized polynomials" in $\beta(0, \cdot)$ and determine which of these functions are in $\mathrm{BMO}^{2}(\Omega)$. In Section 3, we show that, for any uniformly continuous $f$ on $\mathbb{R}^{n}$, the heat transform $\tilde{f}^{(t)}$ is Lipschitz for all $t>0$ and $\tilde{f}^{(t)}$ converges uniformly to $f$ as $t \rightarrow 0$. In Section 4 , we prove the analogous result for $f \in \operatorname{UC}(\Omega)$ when $\Omega$ is any BSD. In Section 5, we give sharper variants of the results in Sections 3 and 4, under a natural restriction. In Sections 3 and 4 we also provide estimates on the Lipschitz constants which allow us to study their asymptotic behaviour. In particular, these estimates can be written in a more explicit form under the stronger assumptions of Section 5.

\section{Uniform continuity and BMO}

We prove two lemmas and a theorem which provide some new perspective on $\operatorname{UC}(\Omega)$.

Lemma 2.1. The class $\mathrm{UC}(\Omega)$ is contained in $\mathrm{BO}(\Omega)$ and in $\mathrm{BMO}^{2}(\Omega)$ for a $B S D \Omega$ or $\Omega=\mathbb{C}^{n}$.

Proof. We use the completeness of the Bergman metric in a standard way to show that $\mathrm{UC}(\Omega)$ is contained in $\mathrm{BO}(\Omega)$. The rest follows from the decomposition (1.3) which can be found in [1,4]. It follows from the completeness of the Bergman metric [9] that for any $z, w$ in $\Omega$, there is a geodesic segment $\gamma$ in $\Omega$ of Bergman arclength $\beta(z, w)$ joining $z$ to $w$. For $f$ in $\operatorname{UC}(\Omega)$, there is a $\delta(f, 1)>0$ so that for $a, b$ in $\Omega$ with $\beta(a, b)<\delta(f, 1)$ we must have $|f(a)-f(b)|<1$. Let $N$ be the greatest integer in $\beta(z, w) \delta^{-1}(f, 1)$ and divide $\gamma$ into $N+1$ segments of equal Bergman arclength. An easy application of the triangle inequality shows that

$$
|f(z)-f(w)|<N+1 \leq \beta(z, w) \delta^{-1}(f, 1)+1 .
$$

Hence, $f \in \mathrm{BO}(\Omega)$ and so $f \in \mathrm{BMO}^{2}(\Omega)$.

Remark 2.2. Lemma 2.1 provides a large number of examples of unbounded functions in $\mathrm{BMO}^{2}(\Omega)$ and seems not to have been noted earlier. In particular, if $f$ is any function in $\operatorname{UC}(\Omega)$ and $g$ is any uniformly continuous function from range $(f)$ into $\mathbb{C}$, then $g \circ f$ is in $\operatorname{UC}(\Omega)$. This shows, for example, that

$$
f(z)=\sqrt{\sqrt{\beta(0, z)}+\beta(0, z)}
$$

is in $\mathrm{UC}(\Omega)$.

For real $\alpha \geq 0$, we now give some useful estimates on the Berezin transform of $\beta(0, z)^{\alpha}$. We write " $\int \cdots d v$ " but understand that, for $\Omega=\mathbb{C}^{n}$, we integrate " $d \mu$ ".

Lemma 2.3. For $B S D(\Omega, d v)$ or $\left(\mathbb{C}^{n}, d \mu\right)$, real $\alpha \geq 0$ and $f_{\alpha}(z)=\beta(0, z)^{\alpha}$, we have

$$
\frac{1}{2^{\alpha}} \beta(0, w)^{\alpha}-K(\alpha) \leq \tilde{f}_{\alpha}(w) \leq 2^{\alpha}\left(\beta(0, w)^{\alpha}+K(\alpha)\right),
$$

where

$$
K(\alpha):=\int_{\Omega} \beta(0, z)^{\alpha} d v(z)=\tilde{f}_{\alpha}(0)
$$


Proof. We use the elementary inequality

$$
(s+t)^{\alpha} \leq\{2 \max (s, t)\}^{\alpha} \leq 2^{\alpha}\left(s^{\alpha}+t^{\alpha}\right)
$$

for $s, t, \alpha \geq 0$. By the invariance of $\beta(\cdot, \cdot)$ under the involutive automorphisms $\varphi_{w}$, we see that

$$
\tilde{f}_{\alpha}(w)=\int_{\Omega} \beta\left(0, \varphi_{w} z\right)^{\alpha} d v(z)=\int_{\Omega} \beta(w, z)^{\alpha} d v(z) .
$$

Now, we estimate

$$
\beta(w, z)^{\alpha} \leq(\beta(w, 0)+\beta(0, z))^{\alpha} \leq 2^{\alpha}\left(\beta(w, 0)^{\alpha}+\beta(0, z)^{\alpha}\right)
$$

and

$$
\beta(w, 0)^{\alpha} \leq(\beta(w, z)+\beta(z, 0))^{\alpha} \leq 2^{\alpha}\left(\beta(w, z)^{\alpha}+\beta(z, 0)^{\alpha}\right) .
$$

Integrating both inequalities with respect to $d v(z)$ or $d \mu(z)$ gives the desired result.

We can now give a complete analysis of membership in $\mathrm{BMO}^{2}(\Omega)$ for generalized polynomials in $\beta(0, z)$. Related results for polynomials on $\mathbb{C}^{n}$ were obtained in [1].

Theorem 2.4. On BSDs $\Omega$ or $\Omega=\mathbb{C}^{n}$ (with measure $d \mu$ ) consider all generalized polynomials in $\beta(0, z)$ of the form

$$
f(z)=\sum_{k=0}^{m} b_{k} \beta(0, z)^{\alpha_{k}},
$$

with $b_{k}$ in $\mathbb{C}, b_{m} \neq 0, \alpha_{k}$ real, $0 \leq \alpha_{k}<\alpha_{k+1}$. The function $f$ is in $\mathrm{BMO}^{2}(\Omega)$ for $\alpha_{m} \leq 1$ and not in $\mathrm{BMO}^{2}(\Omega)$ for $\alpha_{m}>1$.

Proof. First, using the standard inequality $(s+t)^{\alpha} \leq s^{\alpha}+t^{\alpha}$ for $s, t \geq 0$ and $0 \leq \alpha \leq 1$, we see that

$$
\beta(w, 0)^{\alpha} \leq(\beta(w, z)+\beta(z, 0))^{\alpha} \leq \beta(w, z)^{\alpha}+\beta(z, 0)^{\alpha}
$$

so that, by symmetry,

$$
\beta(w, z)^{\alpha} \geq\left|\beta(0, w)^{\alpha}-\beta(0, z)^{\alpha}\right|
$$

and $\beta(0, \cdot)^{\alpha}$ is $\operatorname{UC}(\Omega)$ for all $0 \leq \alpha \leq 1$. It follows from Lemma 2.1 that $f(z)$ is in $\operatorname{BMO}^{2}(\Omega)$ for $\alpha_{m} \leq 1$.

For $\alpha_{m}>1$, we recall that for $f$ to be in $\operatorname{BMO}^{2}(\Omega), \tilde{f}$ must be Lipschitz [1,4]. It follows easily that, for some $C>0$, we must have $C+C \beta(0, w) \geq|\tilde{f}(w)|$. For $f_{\alpha}(z)=\beta(0, z)^{\alpha}$ as in Lemma 2.3, we have

$$
\tilde{f}(w)=b_{m} \tilde{f}_{\alpha_{m}}(w)-\sum_{k=0}^{m-1}\left(-b_{k}\right) \tilde{f}_{\alpha_{k}}(w) .
$$

Thus, using the triangle inequality and Lemma 2.3 , we have

$$
\begin{aligned}
|\tilde{f}(w)| & \geq\left|b_{m}\right| \tilde{f}_{\alpha_{m}}(w)-\sum_{k=0}^{m-1}\left|b_{k}\right| \tilde{f}_{\alpha_{k}}(w) \\
& \geq\left|b_{m}\right|\left(\frac{1}{2^{\alpha_{m}}} f_{\alpha_{m}}(w)-K\left(\alpha_{m}\right)\right)-\sum_{k=0}^{m-1}\left|b_{k}\right| 2^{\alpha_{k}}\left(f_{\alpha_{k}}(w)+K\left(\alpha_{k}\right)\right) .
\end{aligned}
$$


It follows that

(*) $C+C f_{1}(w)+\left|b_{m}\right| K\left(\alpha_{m}\right)+\sum_{k=0}^{m-1}\left|b_{k}\right| 2^{\alpha_{k}}\left(f_{\alpha_{k}}(w)+K\left(\alpha_{k}\right)\right) \geq \frac{1}{2^{\alpha_{m}}}\left|b_{m}\right| f_{\alpha_{m}}(w)$.

Now choose $\varepsilon>0$ so that $\alpha_{m}-\varepsilon>\max \left(\alpha_{m-1}, 1\right)$. Dividing both sides of $(*)$ by $\beta(0, w)^{\alpha_{m}-\varepsilon}$ and taking $\beta(0, w)$ large shows that $f$ cannot be in $\mathrm{BMO}^{2}(\Omega)$ for $\alpha_{m}>1$.

Remark 2.5. It is not hard to check that, for $0 \leq \alpha<1, \beta(0, \cdot)^{\alpha}$ is, in fact, in $\mathrm{VMO}^{2}(\Omega)$.

\section{Real analytic Lipschitz approximation on Euclidean space}

Consider the complex $n$-space $\mathbb{C}^{n}$ equipped with the Gaussian measure $d \mu$. We generalize our notion of the Berezin transform in (1.2) by inserting a positive parameter $t$

$$
\tilde{f}^{(t)}(w):=\frac{1}{(4 \pi t)^{n}} \int_{\mathbb{C}^{n}} f(w-z) e^{-\frac{|z|^{2}}{4 t}} d v(z) .
$$

Note that for each $t>0$ (cf. [2,6]) the integral transform (3.1) in fact is the Berezin transform of $f$ with respect to the new Gaussian measure

$$
d \mu_{t}(z)=\frac{1}{(4 \pi t)^{n}} \exp \left\{-\frac{|z|^{2}}{4 t}\right\} d v(z)
$$

Moreover, note that with our former notation we have $d \mu=d \mu_{1 / 4}$ and $\tilde{f}=\tilde{f}^{\left(\frac{1}{4}\right)}$. The family $\left\{\tilde{f}^{(t)}\right\}_{t>0}$ frequently is called the heat transform of $f$ and due to its relation to the heat operator on $\mathbb{C}^{n} \cong \mathbb{R}^{2 n}$ it fulfills the semi-group property

$$
\left\{\tilde{f}^{(s)}\right\}^{(t)}=\tilde{f}^{(s+t)}
$$

whenever $s, t>0$ are sufficiently small (see [2,6] for more details). It easily can be checked that for all $f \in \mathrm{UC}\left(\mathbb{C}^{n}\right)$ the heat transforms $\tilde{f}^{(t)}(z)$ exist for all $t>0$ and define a semigroup of real analytic functions on $\mathbb{C}^{n}$. As for the next result, we also refer to the recent book [16, Theorem 3.35, p. 127].

Proposition 3.1. For all $t>0$ and with $f \in \mathrm{UC}\left(\mathbb{C}^{n}\right)$ we have $\tilde{f}^{(t)} \in \operatorname{Lip}\left(\mathbb{C}^{n}\right)$ and $f-\tilde{f}^{(t)} \in \operatorname{BUC}\left(\mathbb{C}^{n}\right)$.

Proof. Let $f \in \mathrm{BMO}^{2}\left(\mathbb{C}^{n}\right)$. Then the following Lipschitz estimate has been shown in [1, Corollary 2.7] for all $z, w \in \mathbb{C}^{n}$,

$$
|\tilde{f}(z)-\tilde{f}(w)|=\left|\tilde{f}^{\left(\frac{1}{4}\right)}(z)-\tilde{f}^{\left(\frac{1}{4}\right)}(w)\right| \leq 2\|f\|_{\mathrm{BMO}}|z-w| .
$$

By a change of variables one can check that the heat transforms $\tilde{f}^{(t)}(z)$ for different values of $t$ and with $z \in \mathbb{C}^{n}$ are simply related by

$$
\left\{f(\cdot \sqrt{t}) \widetilde{\}}^{(s)}(z)=\tilde{f}^{(t s)}(z \sqrt{t}) .\right.
$$

Let $f \in \mathrm{UC}\left(\mathbb{C}^{n}\right)$. Then we have for all $t>0$,

$$
f(\cdot \sqrt{t}) \in \mathrm{UC}\left(\mathbb{C}^{n}\right) \subset \mathrm{BMO}^{2}\left(\mathbb{C}^{n}\right) .
$$


Combining (3.3) and (3.4) gives

$$
\begin{aligned}
\left|\tilde{f}^{(t)}(z)-\tilde{f}^{(t)}(w)\right| & =\left|\{f(\cdot 2 \sqrt{t})\}\left(\frac{z}{2 \sqrt{t}}\right)-\{f(\cdot 2 \sqrt{t})\}\left(\frac{w}{2 \sqrt{t}}\right)\right| \\
& \leq t^{-\frac{1}{2}}\|f(\cdot 2 \sqrt{t})\|_{\text {Вмо }}|z-w| .
\end{aligned}
$$

Hence, we have shown that $\tilde{f}^{(t)} \in \operatorname{Lip}\left(\mathbb{C}^{n}\right)$ for all $t>0$.

It is clear that $g_{t}:=f-\tilde{f}^{(t)} \in \mathrm{UC}\left(\mathbb{C}^{n}\right)$ and it remains to show the boundedness of $g_{t}$. Since $\mathrm{UC}\left(\mathbb{C}^{n}\right) \subset \mathrm{BO}\left(\mathbb{C}^{n}\right)$, we can find $C>0$ with $|f(z)-f(w)| \leq C(1+|z-w|)$. It follows for $w \in \mathbb{C}^{n}$ that

$$
\begin{aligned}
\left|g_{t}(w)\right| & \leq \frac{1}{(4 \pi t)^{n}} \int_{\mathbb{C}^{n}}|f(w)-f(w-z)| e^{-\frac{|z|^{2}}{4 t}} d v(z) \\
& \leq \frac{C}{(4 \pi t)^{n}} \int_{\mathbb{C}^{n}}(1+|z|) e^{-\frac{|z|^{2}}{4 t}} d v(z)<\infty
\end{aligned}
$$

Hence we have proved the proposition.

With $f \in \mathrm{UC}\left(\mathbb{C}^{n}\right)$ we consider the limit behaviour of $\tilde{f}^{(t)}$ as $t \rightarrow 0$. A corresponding result in the case of a general bounded symmetric domain $\Omega$ in $\mathbb{C}^{n}$ can be found in Proposition 4.4 below.

Proposition 3.2. Let $f \in \mathrm{UC}\left(\mathbb{C}^{n}\right)$. Then $\lim _{t \rightarrow 0} \tilde{f}^{(t)}=f$ uniformly on $\mathbb{C}^{n}$.

Proof. Since $\mathrm{UC}\left(\mathbb{C}^{n}\right) \subset \mathrm{BO}\left(\mathbb{C}^{n}\right)$, we have $|f(z)-f(w)| \leq K_{f}(1+|z-w|)$ for all $z, w \in \mathbb{C}^{n}$ and with a constant $K_{f}>0$. Moreover, for any $\varepsilon>0$, there is a $\delta=\delta(\varepsilon, f)>0$ so that $|f(z)-f(w)|<\frac{\varepsilon}{2}$ whenever $|z-w|<\delta$. Now consider

$$
\tilde{f}^{(t)}(w)-f(w)=\frac{1}{(4 \pi t)^{n}} \int_{\mathbb{C}^{n}}[f(w-z)-f(w)] e^{-\frac{|z|^{2}}{4 t}} d v(z)
$$

and let $a=z /(2 \sqrt{t})$ so that

$$
\tilde{f}^{(t)}(w)-f(w)=\frac{1}{\pi^{n}} \int_{\mathbb{C}^{n}}[f(w-2 a \sqrt{t})-f(w)] e^{-|a|^{2}} d v(a) .
$$

For $|a|<\delta /(2 \sqrt{t})$ we have $|f(w)-f(w-2 a \sqrt{t})|<\frac{\varepsilon}{2}$ so that

$$
\begin{aligned}
\left|\tilde{f}^{(t)}(w)-f(w)\right| & <\frac{\varepsilon}{2}+\frac{K_{f}}{\pi^{n}} \int_{\{a:|a| \geq \delta /(2 \sqrt{t})\}}(1+2|a| \sqrt{t}) e^{-|a|^{2}} d v(a) \\
& =\frac{\varepsilon}{2}+\frac{K_{f}}{\pi^{n}} I(t) .
\end{aligned}
$$

One observes that $\lim _{t \rightarrow 0} I(t)=0$. Finally, by choosing sufficiently small $t>0$, we see that $\left|\tilde{f}^{(t)}(w)-f(w)\right|<\varepsilon$ for all $w \in \mathbb{C}^{n}$.

The proofs of Propositions 3.1 and 3.2 "descend" from $\Omega=\mathbb{C}^{n}$ to real $n$-space $\mathbb{R}^{n}$. If $f \in \mathrm{UC}\left(\mathbb{R}^{n}\right)$ and $t>0$, then similarly to (3.1) we define

$$
\tilde{f}^{(t)}(u)=\frac{1}{(4 \pi t)^{\frac{n}{2}}} \int_{\mathbb{R}^{n}} f(u-x) e^{-\frac{|x|^{2}}{4 t}} d v(x),
$$

where now $d v$ means the usual Lebesgue measure on $\mathbb{R}^{n}$. 
Corollary 3.3. For $f$ in $\mathrm{UC}\left(\mathbb{R}^{n}\right)$ and all $t>0$, we have $\tilde{f}^{(t)} \in \operatorname{Lip}\left(\mathbb{R}^{n}\right)$ and $f-\tilde{f}^{(t)}$ is in $\mathrm{BUC}\left(\mathbb{R}^{n}\right)$. Moreover, with $u \in \mathbb{R}^{n}$ it holds

$$
\lim _{t \rightarrow 0} \tilde{f}^{(t)}(u)=f(u)
$$

and the convergence is uniform on $\mathbb{R}^{n}$.

Proof. For $z=(x, y)$ with $x, y$ in $\mathbb{R}^{n}$, define $F(x, y)=f(x)$. We see that $F$ is in $\mathrm{UC}\left(\mathbb{C}^{n}\right)$ and can now apply Proposition 3.1. Note that

$$
\begin{aligned}
\widetilde{F}^{(t)}(u, 0) & =(4 \pi t)^{-n} \int_{\mathbb{R}^{n}} \int_{\mathbb{R}^{n}} F(u-x,-y) e^{-\frac{|x|^{2}+|y|^{2}}{4 t}} d v(x) d v(y) \\
& =(4 \pi t)^{-\frac{n}{2}} \int_{\mathbb{R}^{n}} f(u-x) e^{-\frac{|x|^{2}}{4 t}} d v(x) \\
& =\tilde{f}^{(t)}(u),
\end{aligned}
$$

so $\tilde{f}^{(t)}$ is in $\operatorname{Lip}\left(\mathbb{R}^{n}\right)$. Moreover, $F(u, 0)-\widetilde{F}^{(t)}(u, 0)$ is bounded, so $f-\tilde{f}^{(t)}$ is in $\operatorname{BUC}\left(\mathbb{R}^{n}\right)$. We immediately conclude from Proposition 3.2 that

$$
\lim _{t \rightarrow 0} \tilde{f}^{(t)}(u)=\lim _{t \rightarrow 0} \widetilde{F}^{(t)}(u, 0)=F(u, 0)=f(u)
$$

and the convergence is uniform on $\mathbb{R}^{n}$.

Now we sum up our previous results:

Theorem 3.4. Let $f \in \mathrm{UC}\left(\mathbb{C}^{n}\right)$. Then the heat transforms $\left\{\tilde{f}^{(t)}\right\}_{t>0}$ define a flow of real analytic functions in $\operatorname{Lip}\left(\mathbb{C}^{n}\right)$ with

$$
\lim _{t \rightarrow 0} \tilde{f}^{(t)}=f
$$

uniformly on $\mathbb{C}^{n}$. The Lipschitz constant of $\tilde{f}^{(t)}$ is dominated by $C_{t}:=t^{-\frac{1}{2}}\|f(\cdot 2 \sqrt{t})\|_{\mathrm{BMO}}$. In particular, the inclusion $\operatorname{Lip}\left(\mathbb{C}^{n}\right) \subset \mathrm{UC}\left(\mathbb{C}^{n}\right)$ is dense.

Remark 3.5. We point out that the density with respect to the uniform topology of the inclusion $\operatorname{Lip}\left(\mathbb{C}^{n}\right) \subset \mathrm{UC}\left(\mathbb{C}^{n}\right)$ had been known before and can be proven in a more abstract framework, cf. [5]. However, Theorem 3.4 provides a very natural approximation of uniformly continuous functions by real analytic Lipschitz functions via the heat flow and with an explicit control of the Lipschitz constants.

Remark 3.6. Proposition 3.1 is not obvious. It gives an explicit additive decomposition

$$
\mathrm{UC}\left(\mathbb{C}^{n}\right)=\operatorname{Lip}\left(\mathbb{C}^{n}\right)+\operatorname{BUC}\left(\mathbb{C}^{n}\right)
$$

and shows that for $f$ unbounded in $\mathrm{UC}, \tilde{f}$ is also unbounded.

Remark 3.7. There are many interesting examples of Lipschitz functions on BSD $\Omega$. In particular, by [14], if $f$ is in the Bloch space of analytic functions on $\Omega$, then $f$ is Lipschitz. However, as pointed out in [1], the only entire functions in $\mathrm{BMO}^{2}\left(\mathbb{C}^{n}\right)$ are linear functions. 


\section{Real analytic Lipschitz approximation on BSDs}

Let $\Omega \subset \mathbb{C}^{n}$ be an irreducible bounded symmetric domain (BSD) of type $(r, a, b)$ and genus $p=(r-1) a+b+2$ in the Harish-Chandra realization (cf. [8,9]). Let $d v$ be the usual Lebesgue measure on $\mathbb{C}^{n} \cong \mathbb{R}^{2 n}$ restricted to $\Omega$. Recall that to such a domain $\Omega$ one can assign the so-called Jordan triple determinant $h=h(z, w)$ on $\mathbb{C}^{n} \times \mathbb{C}^{n}$ which is a polynomial with respect to the variables $z$ and $\bar{w}$ (see $[8,11,15]$ for details). Moreover, $h$ satisfies

(i) $h(z, 0)=1$ and $h(z, w)=\overline{h(w, z)}$ for all $z, w \in \mathbb{C}^{n}$.

(ii) $h(z, z)>0$ for all $z \in \Omega$ and $h(z, z)=0$ for all $z \in \partial \Omega$.

With a given weight parameter $\lambda>p-1$ we write $L_{\mathrm{an}, \lambda}^{2}(\Omega, d v)$ for the Hilbert space of holomorphic functions $f$ on $\Omega$ such that

$$
\|f\|_{\lambda}^{2}:=c_{\lambda} \int_{\Omega}|f(z)|^{2} h(z, z)^{\lambda-p} d v(z)<\infty .
$$

The constant $c_{\lambda}>0$ is chosen with $\|e\|_{\lambda}=1$ where $e \equiv 1$. It has the explicit form (cf. [8])

$$
c_{\lambda}=\frac{1}{\pi^{n}} \frac{\Gamma_{\Omega}(\lambda)}{\Gamma_{\Omega}\left(\lambda-\frac{n}{r}\right)},
$$

where $\Gamma_{\Omega}(\lambda)$ denotes the Gindikin Gamma function

$$
\Gamma_{\Omega}(\lambda):=(2 \pi)^{\frac{n_{1}-r}{2}} \prod_{j=1}^{r} \Gamma\left(\lambda-(j-1) \frac{a}{2}\right) \quad \text { with } \quad n_{1}:=\frac{r(r-1)}{2} a+r .
$$

We call $L_{\text {an }, \lambda}^{2}(\Omega, d v)$ the standard weighted Bergman space over $\Omega$. As is well known [8], the reproducing kernel $K_{\lambda}: \Omega \times \Omega \rightarrow \mathbb{C}$ of $L_{\text {an, } \lambda}^{2}(\Omega, d v)$ is related to $h$ via

$$
K_{\lambda}(z, w)=h(z, w)^{-\lambda} \text {. }
$$

For a given function $g \in L^{1}(\Omega, d v)$ and with $\lambda \geq p$ consider the integral transform

$$
B_{\lambda}(g)(w):=c_{\lambda} \int_{\Omega} g \circ \varphi_{w}(z) h(z, z)^{\lambda-p} d v(z) .
$$

On the right-hand side we write $\varphi_{w}$ for the (unique up to unitary multiples) automorphism of $\Omega$ with $\varphi_{w} \circ \varphi_{w}=\mathrm{id}$ and $\varphi_{w}(0)=w$. We will show below that the integral (4.3) exists. Note that according to (1.1) and with $\beta(\cdot, 0) \in L^{p}(\Omega, d v)$ for all $p>1$ we have

$$
\mathrm{UC}(\Omega) \subset \mathrm{BO}(\Omega) \subset L^{1}(\Omega, d v) .
$$

In particular, the integral transform $B_{\lambda}(g)$ is well defined for all $g \in \operatorname{UC}(\Omega)$.

We give another expression for $B_{\lambda}(g)(w)$. Let $J_{\mathbb{C}} \varphi_{w}$ denote the complex Jacobian of $\varphi_{w}(z)$ for fixed $w \in \Omega$. From the well-known transformation rule of the (unweighted) Bergman kernel together with (4.2) we obtain

$$
\begin{aligned}
h\left(z_{1}, z_{2}\right)^{-p} & =K_{p}\left(z_{1}, z_{2}\right) \\
& =K_{p}\left(\varphi_{w} z_{1}, \varphi_{w} z_{2}\right) J_{\mathbb{C}} \varphi_{w}\left(z_{1}\right) \overline{J_{\mathbb{C}} \varphi_{w}\left(z_{2}\right)} \\
& =h\left(\varphi_{w} z_{1}, \varphi_{w} z_{2}\right)^{-p} J_{\mathbb{C}} \varphi_{w}\left(z_{1}\right) \overline{J_{\mathbb{C}} \varphi_{w}\left(z_{2}\right)} .
\end{aligned}
$$


In particular, if we chose $w=z_{2}$ and $w=z_{1}=z_{2}$, then the last equation and the property (i) of $h$ imply the identities

$$
\begin{aligned}
h\left(z_{1}, w\right)^{-p} & =J_{\mathbb{C}} \varphi_{w}\left(z_{1}\right) \overline{J_{\mathbb{C}} \varphi_{w}(w)}, \\
h(w, w)^{-p} & =\left|J_{\mathbb{C}} \varphi_{w}(w)\right|^{2} .
\end{aligned}
$$

We find

$$
J_{\mathbb{C}} \varphi_{w}\left(z_{1}\right)=h\left(z_{1}, w\right)^{-p} \overline{J_{\mathbb{C}} \varphi_{w}(w)^{-1}}=e_{w} \cdot h\left(z_{1}, w\right)^{-p} h(w, w)^{\frac{p}{2}}
$$

with some constant $e_{w} \in \mathbb{C}$ depending only on $w \in \Omega$ and with $\left|e_{w}\right|=1$. Using this relation in formula (4.4) with $z_{1}=z_{2}=z \in \Omega$ shows that

$$
h\left(\varphi_{w} z, \varphi_{w} z\right)=\frac{h(z, z) h(w, w)}{|h(z, w)|^{2}} .
$$

Now, using the transformation rule of the integral in (4.3) gives

$$
\begin{aligned}
B_{\lambda}(g)(w) & =c_{\lambda} \int_{\Omega}\left|J_{\mathbb{C}} \varphi_{w}(z)\right|^{2} g(z) h\left(\varphi_{w} z, \varphi_{w} z\right)^{\lambda-p} d v(z) \\
& =c_{\lambda} \int_{\Omega} g(z) \frac{|h(z, w)|^{-2 \lambda}}{h(w, w)^{-\lambda}} h(z, z)^{\lambda-p} d v(z) .
\end{aligned}
$$

In particular, it follows from the boundedness of $h(\cdot, w)^{-\lambda}$ for all $w \in \Omega$ (cf. [8, Theorem 3.8]) that $B_{\lambda}(g)(w)$ is well-defined for all $g \in L^{1}(\Omega, d v)$ and $\lambda \geq p$.

Consider now the normalized reproducing kernel $k_{w}^{\lambda}$ of the weighted Bergman space $L_{\text {an }, \lambda}^{2}(\Omega, d v)$ given by

$$
k_{w}^{\lambda}(z):=\frac{K_{\lambda}(z, w)}{\left\|K_{\lambda}(\cdot, w)\right\|_{\lambda}}=\frac{h(z, w)^{-\lambda}}{h(w, w)^{-\frac{\lambda}{2}}}, \quad z, w \in \Omega .
$$

Then we can rewrite (4.6) in the form

$$
B_{\lambda}(g)(w)=c_{\lambda} \int_{\Omega} g(z)\left|k_{w}^{\lambda}(z)\right|^{2} h(z, z)^{\lambda-p} d v(z) .
$$

Lemma 4.1. Let $g \in L^{1}(\Omega, d v)$ and $\lambda \geq p$. Then $B_{\lambda}(g)$ is real analytic on $\Omega$.

Proof. Let $v \in \Omega$. Then it is sufficient to show that the function

$$
G(v):=\int_{\Omega} g(z)|h(z, v)|^{-2 \lambda} h(z, z)^{\lambda-p} d v(z)
$$

is real analytic in a neighbourhood $U \subset \Omega$ of $v$ such that $\bar{U} \subset \Omega$, where $\bar{U}$ denotes the closure of $U$. Note that $G$ is the restriction to $\Delta:=\left\{(v, \bar{v}) \subset \Omega^{2}: v \in \Omega\right\}$ of the function

$$
\widetilde{G}(v, w):=\int_{\Omega} g(z) h(z, \bar{w})^{-\lambda} h(v, z)^{-\lambda} h(z, z)^{\lambda-p} d v(z) .
$$

We show that $\widetilde{G}$ is holomorphic in the neighbourhood $\widetilde{U}:=\left\{\left(v_{1}, \overline{v_{2}}\right) \in \Omega^{2}: v_{1}, v_{2} \in U\right\}$, which will prove the lemma. Consider the standard expansion of the reproducing kernel

$$
h(z, w)^{-\lambda}=\sum_{\mathbf{m} \in M}(\lambda)_{\mathbf{m}} K^{\mathbf{m}}(z, w),
$$

where $M$ denotes the set of all tuples $\mathbf{m}=\left(m_{1}, \ldots, m_{r}\right) \in \mathbb{N}_{0}^{r}$ with $m_{1} \geq \cdots \geq m_{r} \geq 0$ and $(\lambda)_{\mathbf{m}}$ is the generalized Pochhammer symbol in [8]. 
For fixed $w \in \mathbb{C}^{n}$ the functions $K^{\mathbf{m}}(z, w)$ are certain complex analytic polynomials homogeneous of degree $|\mathbf{m}|=m_{1}+\cdots+m_{r}$ in $z \in \mathbb{C}^{n}$ and it holds $K^{\mathbf{m}}(z, w)=\overline{K^{\mathbf{m}}(w, z)}$. It is known (cf. [8, Theorem 3.8]) that the convergence in (4.9) is uniform and absolute on compact subsets of $\Omega \times \bar{\Omega}$. Hence we can interchange integration and summation in (4.8). With $(v, w) \in \widetilde{U}$ we have

$$
\widetilde{G}(v, w)=\sum_{\mathbf{m}, \mathbf{q} \in M}(\lambda)_{\mathbf{m}}(\lambda)_{\mathbf{q}} \int_{\Omega} g(z) K^{\mathbf{m}}(z, \bar{w}) K^{\mathbf{q}}(v, z) h^{\lambda-p}(z, z) d v(z) .
$$

Since $\lambda \geq p$, the integrals on the right-hand side exist and for all $\mathbf{m}, \mathbf{q} \in M$ they define holomorphic polynomials in $(v, w)$ homogeneous of degree $|\mathbf{m}|+|\mathbf{q}|$. It follows that $\widetilde{G}(v, w)$ is holomorphic in $\widetilde{U}$.

In the next example, we calculate $B_{\lambda}$ in the case of a rank one domain more explicitly.

Example 4.2. Let $\Omega=\mathbb{B}^{n}$ be the Euclidean unit ball in $\mathbb{C}^{n}$. Then we have $r=1$ and $p=n+1$. In this case, the Gindikin Gamma function $\Gamma_{\Omega}(\lambda)$ coincides with the usual Gamma function $\Gamma(\lambda)$. Thus (4.3) with $\lambda:=n+1+\alpha$ and $\alpha \geq 0$ takes the form

$$
\begin{aligned}
B_{n+1+\alpha}(g)(w) & =\frac{1}{\pi^{n}} \frac{\Gamma(n+1+\alpha)}{\Gamma(\alpha+1)} \int_{\mathbb{B}^{n}} g \circ \varphi_{w}(z)\left(1-|z|^{2}\right)^{\alpha} d v(z) \\
& =\frac{1}{\pi^{n}} \frac{\Gamma(n+1+\alpha)}{\Gamma(\alpha+1)} \int_{\mathbb{B}^{n}} g(z) \frac{\left(1-|w|^{2}\right)^{n+1+\alpha}\left(1-|z|^{2}\right)^{\alpha}}{|1-z \cdot w|^{2(n+1+\alpha)}} d v(z),
\end{aligned}
$$

where $g \in L^{1}\left(\mathbb{B}^{n}, d v\right)$. Recall that in the literature the expression $B_{n+1+\alpha}(g)$ is also called the $\alpha$-Berezin transform of $g$ (cf. $[12,13]$ ).

Let $\operatorname{Aut}_{0}(\Omega)$ denote the connected component of the automorphism group of $\Omega$ characterized by $\operatorname{Id} \in \operatorname{Aut}_{0}(\Omega)$. We write $K \subset \operatorname{Aut}_{0}(\Omega)$ for the sub-group of all automorphisms leaving the origin $0 \in \Omega$ invariant. As is well known, there is a set $\left\{e_{1}, \ldots, e_{r}\right\} \subset \mathbb{C}^{n}$ of $\mathbb{R}$-linear independent vectors (Jordan frame) such that each $z \in \mathbb{C}^{n}$ has a polar decomposition of the form

$$
z=k \sum_{j=1}^{r} t_{j} e_{j} \quad \text { with } \quad k \in K, t_{1}, \ldots, t_{r} \in \mathbb{R} .
$$

Moreover, $z \in \Omega$ if and only if $\left|t_{j}\right|<1$ for all $j=1, \ldots, r$.

Lemma 4.3. Assume that $g \in L^{1}(\Omega, d v)$ identically vanishes in an open ball $\mathbb{B}_{\delta}^{n} \subset \Omega$ of radius $\delta>0$ and centered at the origin. Then

$$
\lim _{\lambda \rightarrow \infty} c_{\lambda} \int_{\Omega} g(z) h(z, z)^{\lambda-p} d v(z)=0 .
$$

Proof. If $z \in \Omega$ is expressed in the polar decomposition (4.10), then the Jordan triple determinant restricted to the diagonal can be written as

$$
h(z, z)=\prod_{j=1}^{r}\left(1-t_{j}^{2}\right) .
$$


In particular, we see that $h(z, z) \leq 1$ and $h(z, z)=1$ if and only if $z=0 \in \Omega$. Pick $0<\rho<1$ with $0<h(z, z)<\rho$ for all $z \in \Omega \backslash \mathbb{B}_{\delta}^{n}$. Then we obtain for $z \in \Omega \backslash \mathbb{B}_{\delta}^{n}$ and with a sufficiently large weight parameter $\lambda \geq p$,

$$
\begin{aligned}
c_{\lambda} h(z, z)^{\lambda-p} & \leq \frac{\rho^{\lambda-p}}{\pi^{n}} \cdot \frac{\Gamma_{\Omega}(\lambda)}{\Gamma_{\Omega}(\lambda-n / r)} \\
& \leq \frac{\rho^{\lambda-p}}{\pi^{n}} \cdot \prod_{j=1}^{r} \frac{\Gamma\left(\lambda-(j-1) \frac{a}{2}\right)}{\Gamma\left(\lambda-(j-1) \frac{a}{2}-\frac{n}{r}\right)} \\
& \leq \frac{\rho^{\lambda-p}}{\pi^{n}} \cdot \prod_{j=1}^{r} \frac{\Gamma\left(\lambda-(j-1) \frac{a}{2}\right)}{\Gamma\left(\lambda-(j-1) \frac{a}{2}-n\right)} \\
& =\frac{\rho^{\lambda-p}}{\pi^{n}} \cdot \prod_{j=1}^{r} \prod_{k=1}^{n}\left(\lambda-(j-1) \frac{a}{2}-k\right) \\
& \leq \frac{\rho^{\lambda-p}}{\pi^{n}} \cdot \lambda^{n r} .
\end{aligned}
$$

The right-hand side tends to zero as $\lambda \rightarrow \infty$ and the assertion easily follows from the assumption on $g$ and the estimate

$$
\left|c_{\lambda} \int_{\Omega} g(z) h(z, z)^{\lambda-p} d v(z)\right| \leq \frac{\rho^{\lambda-p}}{\pi^{n}} \cdot \lambda^{n r}\|g\|_{L^{1}(\Omega, d v)} .
$$

As before, we write $\beta(\cdot, \cdot)$ for the Bergman metric on $\Omega$. Recall that due to Lemma 2.1 the space $\operatorname{UC}(\Omega)$ is contained in $\operatorname{BO}(\Omega)$. Hence for any $f \in \operatorname{UC}(\Omega)$ there is a $C_{f}>0$ such that for all $z, w \in \Omega$,

$$
|f(z)-f(w)| \leq C[1+\beta(z, w)] .
$$

Now, we can prove a result analogous to the statement of Proposition 3.2:

Proposition 4.4. Let $f \in \mathrm{UC}(\Omega)$. Then $\lim _{\lambda \rightarrow \infty} B_{\lambda}(f)=f$ uniformly on $\Omega$.

Proof. Let $\varepsilon>0$ and choose $\delta>0$ such that $|f(z)-f(w)|<\varepsilon$ whenever $z, w \in \Omega$ with $\beta(z, w)<\delta$. Since the $\beta$-topology is equivalent to the Euclidean topology on $\Omega$ (see [9]), we can pick $\delta^{\prime}$ with $0<\delta^{\prime}<1$ so that $\left\{z \in \Omega:|z|<\delta^{\prime}\right\} \subset\{z \in \Omega: \beta(z, 0)<\delta\}$. If $w \in \Omega$ and $\lambda \geq p$, then we have

$$
\begin{aligned}
\left|B_{\lambda}(f)(w)-f(w)\right| & \leq c_{\lambda} \int_{\Omega}\left|f \circ \varphi_{w}(z)-f \circ \varphi_{w}(0)\right| h(z, z)^{\lambda-p} d v(z) \\
& \leq c_{\lambda}\left\{\int_{|z|<\delta^{\prime}}+\int_{|z| \geq \delta^{\prime}}\right\}\left|f \circ \varphi_{w}(z)-f \circ \varphi_{w}(0)\right| h(z, z)^{\lambda-p} d v(z) \\
& =(*) .
\end{aligned}
$$

Note that in the case where $|z|<\delta^{\prime}$ we also have $\beta\left(\varphi_{w} z, \varphi_{w} 0\right)=\beta(z, 0)<\delta$ and therefore it follows from the uniform continuity of $f$ that

$$
\left|f \circ \varphi_{w}(z)-f \circ \varphi_{w}(0)\right|<\varepsilon .
$$


Hence, using this estimate together with (4.11) shows

$$
(*) \leq \varepsilon+c_{\lambda} C \int_{|z| \geq \delta^{\prime}}[1+\beta(z, 0)] h(z, z)^{\lambda-p} d v(z) .
$$

It is known (see [4, Theorem E]) that the function $\beta(\cdot, 0)$ defines an element in $L^{1}(\Omega, d v)$. Hence we conclude from Lemma 4.3 that

$$
\lim _{\lambda \rightarrow \infty} c_{\lambda} \int_{|z| \geq \delta^{\prime}}[1+\beta(z, 0)] h(z, z)^{\lambda-p} d v(z)=0
$$

and the proposition is proven.

In the case of the Euclidean unit ball $\Omega=\mathbb{B}^{n}$ in $\mathbb{C}^{n}$ the following Lipschitz estimate for the $\alpha$-Berezin transform has been proven in [13, Theorem 2.11] or [12, Lemma 2.14].

Proposition 4.5. Let $\mu$ be a measure on $\mathbb{B}^{n}$ such that $|\mu|$ is Carleson. Then there is a constant $C>0$ such that

$$
\left|B_{n+1+\alpha}(\mu)(z)-B_{n+1+\alpha}(\mu)(w)\right| \leq C\left\|T_{\mu}\right\| \beta(z, w) .
$$

Here $T_{\mu}$ denotes the Toeplitz operator with (measure) symbol $\mu$ acting on $L_{\mathrm{an}, \lambda}^{2}\left(\mathbb{B}^{n}, d v\right)$.

For our purpose in the present paper we need a different Lipschitz estimate for the integral transforms $B_{\lambda}(g)$ which is valid for arbitrary BSDs and also applies to functions $g \in \operatorname{UC}(\Omega)$ (in which case the Toeplitz operator $T_{g}$ might be unbounded even in ball case).

For the rest of the section we have to introduce some new notation.

Definition 4.6. Let $\lambda \geq p$ and $f \in L^{2}(\Omega, d v)$, where $\Omega$ is any BSD. Then the " $\lambda$-mean oscillation" $\mathrm{MO}^{\lambda}(f, z)$ of $f$ is defined by

$$
\operatorname{MO}^{\lambda}(f, z):=B_{\lambda}\left(|f|^{2}\right)(z)-\left|B_{\lambda}(f)(z)\right|^{2} .
$$

We say that $f$ is of "bounded $\lambda$-mean oscillation" on $\Omega$ if $\mathrm{MO}^{\lambda}(f, \cdot)$ defines a bounded function on $\Omega$. Let $\mathrm{BMO}_{\lambda}^{2}(\Omega)$ be the space of all functions of bounded $\lambda$-mean oscillation.

Note that $\mathrm{BO}(\Omega)$ is contained in $\mathrm{BMO}_{\lambda}^{2}(\Omega)$ for all $\lambda \geq p$ by a minor modification of the proof of [4, Theorem 13]. By a straightforward calculation one can check that

$$
\operatorname{MO}^{\lambda}(f, z)=B_{\lambda}\left(\left|f-B_{\lambda}(f)(z)\right|^{2}\right)(z)
$$

and since $B_{\lambda}$ has a non-negative integral kernel, according to (4.7) we conclude that $\operatorname{MO}^{\lambda}(f, \cdot)$ is a non-negative function. Let $S$ be any subset of $\Omega$. Then we write

$$
\|f\|_{\mathrm{BMO}_{\lambda}(S)}:=\sup _{z \in S} \mathrm{MO}^{\lambda}(f, z)^{\frac{1}{2}} \text { and }\|f\|_{\mathrm{BMO}_{\lambda}}:=\|f\|_{\mathrm{BMO}_{\lambda}(\Omega)} .
$$

Note that $\|\cdot\|_{\mathrm{BMO}_{\lambda}}$ defines a semi-norm on $\operatorname{BMO}_{\lambda}^{2}(\Omega)$.

For any given $w \in \Omega$ let $P_{w}^{\lambda}$ denote the rank-one orthogonal projection onto the linear span of the normalized kernel function $k_{w}^{\lambda}$ in $L_{\mathrm{an}, \lambda}^{2}(\Omega, d v)$.

The following result generalizes $[4$, Theorem F] to the case of weighted Bergman spaces. 
Proposition 4.7. Let $\lambda \geq p$ and $\gamma: I:=[0,1] \rightarrow \Omega$ be a smooth curve. For any function $g \in \mathrm{BMO}_{\lambda}^{2}(\Omega)$ we have

$$
\left|\frac{d}{d t}\left\{B_{\lambda}(g) \circ \gamma(t)\right\}\right| \leq 2\|g\|_{\mathrm{BMO}_{\lambda}(\gamma(I))}\left\|\left[I-P_{\gamma(t)}^{\lambda}\right]\left(\frac{d}{d t} k_{\gamma(t)}^{\lambda}\right)\right\|_{\lambda} .
$$

Proof. We express $B_{\lambda}(g)$ in the form (4.7) and differentiate under the integral sign to obtain

$$
\text { (4.15) } \begin{aligned}
\frac{d}{d t}\left\{B_{\lambda}(g) \circ \gamma(t)\right\} & =2 c_{\lambda} \int_{\Omega} g(z) \operatorname{Re}\left\{\left(\frac{d}{d t} k_{\gamma(t)}^{\lambda}(z)\right) \overline{k_{\gamma(t)}^{\lambda}(z)}\right\} h(z, z)^{\lambda-p} d v(z) \\
& =(*) .
\end{aligned}
$$

Another differentiation of the identity $\left\langle k_{\gamma(t)}^{\lambda}, k_{\gamma(t)}^{\lambda}\right\rangle_{\lambda} \equiv 1$ with respect to the $t$-variable shows that

$$
\operatorname{Re}\left\langle\frac{d}{d t} k_{\gamma(t)}^{\lambda}, k_{\gamma(t)}^{\lambda}\right\rangle_{\lambda}=0 .
$$

Therefore we obtain from $P_{\gamma(t)}^{\lambda} f=\left\langle f, k_{\gamma(t)}^{\lambda}\right\rangle_{\lambda} \cdot k_{\gamma(t)}^{\lambda}$ that

$$
\operatorname{Re}\left\{P_{\gamma(t)}^{\lambda}\left(\frac{d}{d t} k_{\gamma(t)}^{\lambda}\right)(z) \overline{k_{\gamma(t)}^{\lambda}(z)}\right\}=\operatorname{Re}\left\{\left\langle\frac{d}{d t} k_{\gamma(t)}^{\lambda}, k_{\gamma(t)}^{\lambda}\right\rangle_{\lambda}\left|k_{\gamma(t)}^{\lambda}(z)\right|^{2}\right\}=0 .
$$

From (4.15) we see that

$$
(*)=2 c_{\lambda} \int_{\Omega} g(z) \operatorname{Re}\left\{\left[I-P_{\gamma(t)}^{\lambda}\right]\left(\frac{d}{d t} k_{\gamma(t)}^{\lambda}(z)\right) \overline{k_{\gamma(t)}^{\lambda}(z)}\right\} h(z, z)^{\lambda-p} d v(z) .
$$

In particular, if we choose $g \equiv 1$, then we have $B_{\lambda}(g) \circ \gamma(t) \equiv 1$ and therefore the last identity gives

$$
\int_{\Omega} \operatorname{Re}\left\{\left[I-P_{\gamma(t)}^{\lambda}\right]\left(\frac{d}{d t} k_{\gamma(t)}^{\lambda}(z)\right) \overline{k_{\gamma(t)}^{\lambda}(z)}\right\} h(z, z)^{\lambda-p} d v(z)=0 .
$$

Now we combine the last two equations to the relation

$$
(*)=2 c_{\lambda} \int_{\mathbb{B}^{n}}\left[g(z)-B_{\lambda}(g) \circ \gamma(t)\right] \operatorname{Re}\left\{\left[I-P_{\gamma(t)}^{\lambda}\right]\left(\frac{d}{d t} k_{\gamma(t)}^{\lambda}(z)\right) \overline{k_{\gamma(t)}^{\lambda}(z)}\right\} h(z, z)^{\lambda-p} d v(z) .
$$

An application of the Cauchy-Schwarz inequality gives

$$
\begin{aligned}
& \left|\frac{d}{d t}\left\{B_{\lambda}(g) \circ \gamma(t)\right\}\right| \\
& \leq 2 c_{\lambda} \int_{\Omega}\left|g(z)-B_{\lambda}(g) \circ \gamma(t)\right|\left|k_{\gamma(t)}^{\lambda}(z)\right| \cdot\left|\left[I-P_{\gamma(t)}^{\lambda}\right]\left(\frac{d}{d t} k_{\gamma(t)}^{\lambda}(z)\right)\right| h(z, z)^{\lambda-p} d v(z) \\
& \leq 2\left\{c_{\lambda} \int_{\Omega}\left|g(z)-B_{\lambda}(g) \circ \gamma(t)\right|^{2}\left|k_{\gamma(t)}^{\lambda}(z)\right|^{2} h(z, z)^{\lambda-p} d v(z)\right\}^{\frac{1}{2}}\left\|\left[I-P_{\gamma(t)}^{\lambda}\right]\left(\frac{d}{d t} k_{\gamma(t)}^{\lambda}\right)\right\|_{\lambda} \\
& =2 \sqrt{B_{\lambda}\left(\left|g-B_{\lambda}(g) \circ \gamma(t)\right|^{2}\right) \circ \gamma(t)} \cdot\left\|\left[I-P_{\gamma(t)}^{\lambda}\right]\left(\frac{d}{d t} k_{\gamma(t)}^{\lambda}\right)\right\|_{\lambda} \cdot
\end{aligned}
$$

Finally, using (4.13) shows the assertion. 
Now we relate the second term on the right-hand side of (4.14) to the Bergman metric $\beta(\cdot, \cdot)$ on $\Omega$. As before, we write $K_{\lambda}(z, w)=h(z, w)^{-\lambda}$ with $z, w \in \Omega$ for the reproducing kernel function of $L_{\text {an }, \lambda}^{2}(\Omega, d v)$. The infinitesimal Bergman metric on $\Omega$ with respect to the parameter $\lambda>p-1$ is given by

$$
G_{z}^{\lambda}=\left(g_{i, j}^{\lambda}(z)\right)_{i, j}=\left(\frac{\partial^{2}}{\partial z_{j} \partial \overline{z_{j}}} \log K_{\lambda}(z, z)\right)_{i, j}=-\lambda\left(\frac{\partial^{2}}{\partial z_{j} \partial \overline{z_{j}}} \log h(z, z)\right)_{i, j} .
$$

It follows that

$$
G_{z}^{\lambda}=\frac{\lambda}{p} \cdot G_{z}^{p},
$$

where $G_{z}^{p}$ is the infinitesimal Bergman metric corresponding to $\beta$ (the unweighted case).

Let $\gamma=\left(\gamma_{1}, \ldots, \gamma_{n}\right): I=[0,1] \rightarrow \Omega$ be a smooth curve with arc length $s_{\lambda}=s_{\lambda}(t)$ with respect to $G_{z}^{\lambda}$. Put $s=s_{p}$. Then we have

$$
\left(\frac{d s_{\lambda}}{d t}\right)^{2}=\sum_{i, j=1}^{n} g_{i, j}^{\lambda}(\gamma(t)) \gamma_{i}^{\prime}(t) \overline{\gamma_{j}^{\prime}(t)}
$$

The following result is analogous to [4, Lemma 1].

Proposition 4.8. For any smooth curve $\gamma$ as above and $\lambda>p-1$ we have

$$
\frac{d s_{\lambda}}{d t}=\left\|\left(I-P_{\gamma(t)}^{\lambda}\right)\left(\frac{d}{d t} k_{\gamma(t)}^{\lambda}\right)\right\|_{\lambda}
$$

Proof. By a direct calculation using $\left(\partial / \partial z_{i}\right) \overline{K_{\lambda}(\cdot, z)}=\overline{\left(\partial / \partial \overline{z_{i}}\right) K_{\lambda}(\cdot, z)}$ one has

$$
\begin{aligned}
\frac{\partial^{2}}{\partial z_{i} \partial \overline{z_{j}}} \log K_{\lambda}(z, z)= & \frac{\partial^{2}}{\partial z_{i} \partial \overline{z_{j}}} \log \left\langle K_{\lambda}(\cdot, z), K_{\lambda}(\cdot, z)\right\rangle_{\lambda} \\
= & K_{\lambda}(z, z)^{-1}\left\langle\frac{\partial}{\partial \overline{z_{j}}} K_{\lambda}(\cdot, z), \frac{\partial}{\partial \overline{z_{i}}} K_{\lambda}(\cdot, z)\right\rangle_{\lambda} \\
& \quad-K_{\lambda}(z, z)^{-1}\left\langle\frac{\partial}{\partial \overline{z_{j}}} K_{\lambda}(\cdot, z), k_{z}^{\lambda}\right\rangle_{\lambda}\left\langle k_{z}^{\lambda}, \frac{\partial}{\partial \overline{z_{i}}} K_{\lambda}(\cdot, z)\right\rangle_{\lambda} .
\end{aligned}
$$

According to our previous notation we can rewrite this identity in the form

$$
\frac{\partial^{2}}{\partial z_{i} \partial \overline{z_{j}}} \log K_{\lambda}(z, z)=K_{\lambda}(z, z)^{-1}\left\langle\left(I-P_{z}^{\lambda}\right) \frac{\partial}{\partial \overline{z_{j}}} K_{\lambda}(\cdot, z), \frac{\partial}{\partial \overline{z_{i}}} K_{\lambda}(\cdot, z)\right\rangle_{\lambda} .
$$

Inserting the expression on the right into (4.17) gives

$$
\left(\frac{d s_{\lambda}}{d t}\right)^{2}=K_{\lambda}(\gamma(t), \gamma(t))^{-1}\left\|\left.\left(I-P_{\gamma(t)}^{\lambda}\right) \sum_{j=1}^{n} \overline{\gamma_{j}^{\prime}(t)} \frac{\partial}{\partial \bar{z}_{j}} K_{\lambda}(\cdot, z)\right|_{z=\gamma(t)}\right\|_{\lambda}^{2} .
$$

Now we use the relations

$$
\frac{\partial}{\partial \overline{z_{j}}} k_{z}^{\lambda}=k_{z}^{\lambda}\left\{K_{\lambda}(z, z)^{\frac{1}{2}} \frac{\partial}{\partial \overline{z_{j}}} K_{\lambda}(z, z)^{-\frac{1}{2}}\right\}+K_{\lambda}^{-\frac{1}{2}}(z, z) \frac{\partial}{\partial \overline{z_{j}}} K_{\lambda}(\cdot, z)
$$

for $j=1, \ldots, n$ which imply that

$$
\left(\frac{d s_{\lambda}}{d t}\right)^{2}=\left\|\left.\left(I-P_{\gamma(t)}^{\lambda}\right) \sum_{j=1}^{n} \frac{\partial}{\gamma_{j}^{\prime}(t)} \frac{\partial}{\partial \overline{z_{j}}} k_{z}^{\lambda}\right|_{z=\gamma(t)}\right\|_{\lambda}^{2} .
$$


Since the derivatives

$$
\frac{\partial}{\partial z_{i}} k_{z}^{\lambda}=k_{z}^{\lambda}\left\{K_{\lambda}(z, z)^{\frac{1}{2}} \frac{\partial}{\partial z_{i}} K_{\lambda}(z, z)\right\}
$$

define elements in the one-dimensional space $\operatorname{span}\left\{k_{z}^{\lambda}\right\} \subset L_{\text {an }, \lambda}^{2}(\Omega, d v)$ and with the total derivative

$$
\frac{d}{d t} k_{\gamma(t)}^{\lambda}=\sum_{i=1}^{n} \gamma_{i}^{\prime}(t) \frac{\partial}{\partial z_{i}} k_{\left.z\right|_{z=\gamma(t)}}^{\lambda}+\sum_{j=1}^{n} \overline{\gamma_{j}^{\prime}(t)} \frac{\partial}{\partial \bar{z}_{j}} k_{\left.z\right|_{z=\gamma(t)}}^{\lambda},
$$

it follows that

$$
\left(I-P_{\gamma(t)}^{\lambda}\right)\left(\frac{d}{d t} k_{\gamma(t)}^{\lambda}\right)=\left(I-P_{\gamma(t)}^{\lambda}\right) \sum_{j=1}^{n} \overline{\gamma_{j}^{\prime}(t)} \frac{\partial}{\partial \overline{z_{j}}} k_{\left.z\right|_{z=\gamma(t)} ^{\lambda}}^{\lambda}
$$

which together with (4.18) implies the assertion.

Let $g \in \mathrm{BMO}_{\lambda}^{2}(\Omega)$. By combining the results of Proposition 4.7 and Proposition 4.8 we obtain

$$
\left|\frac{d}{d t}\left\{B_{\lambda}(g) \circ \gamma(t)\right\}\right| \leq 2\|g\|_{\mathrm{BMO}_{\lambda}}\left(\frac{d s_{\lambda}}{d t}\right) .
$$

Now we get a Lipschitz estimate for $B_{\lambda}(g)$ which holds for all $g \in \operatorname{BMO}_{\lambda}^{2}(\Omega)$ :

Theorem 4.9. Let $g \in \mathrm{BMO}_{\lambda}^{2}(\Omega)$ and $\lambda \geq p$. Then we have for all $z, w \in \Omega$,

$$
\left|B_{\lambda}(g)(w)-B_{\lambda}(g)(z)\right| \leq 2 \sqrt{\frac{\lambda}{p}} \cdot\|g\|_{\mathrm{BMO}_{\lambda}} \beta(z, w) .
$$

In the case where $g \in \mathrm{UC}(\Omega)$, we have $g-B_{\lambda}(g) \in \operatorname{BUC}(\Omega)$.

Proof. Let $\gamma$ be a geodesic joining $z$ and $w$ with respect to the Bergman metric corresponding to the weighted kernel $K_{\lambda}$. Let $\beta_{\lambda}(\cdot, \cdot)$ denote the Bergman distance function. Note that according to (4.16) we have

$$
\beta(z, w)=\sqrt{\frac{\lambda}{p}} \cdot \beta_{\lambda}(z, w) .
$$

The estimate (4.20) follows from this observation and by integrating (4.19). If $g \in \mathrm{UC}(\Omega)$, then it follows from $\operatorname{Lip}(\Omega) \subset \mathrm{UC}(\Omega)$ that $g-B_{\lambda}(g) \in \mathrm{UC}(\Omega)$ for all $\lambda \geq p$. It remains to show the boundedness of $g-B_{\lambda}(g)$. From

$$
g(w)-B_{\lambda}(g)(w)=c_{\lambda} \int_{\Omega}\left[g(w)-g \circ \varphi_{w}(z)\right] h(z, z)^{\lambda-p} d v(z)
$$

we obtain the estimate

$$
\left|g(w)-B_{\lambda}(g)(w)\right| \leq c_{\lambda} \int_{\Omega}\left|g \circ \varphi_{w}(0)-g \circ \varphi_{w}(z)\right| h(z, z)^{\lambda-p} d v(z) .
$$


Since $g \in \mathrm{BO}(\Omega)$ and $\beta\left(\varphi_{w} 0, \varphi_{w} z\right)=\beta(0, z)$, we see from (1.1) and with $C>0$ that

$$
\begin{aligned}
\left|g(w)-B_{\lambda}(g)(w)\right| & \leq c_{\lambda} C \int_{\Omega}[1+\beta(0, z)] h(z, z)^{\lambda-p} d v(z) \\
& \leq c_{\lambda} C \int_{\Omega}[1+\beta(0, z)] d v(z)<\infty .
\end{aligned}
$$

In the last line we have used $\lambda \geq p$ and $0<h(z, z) \leq 1$ for all $z \in \Omega$ together with the wellknown fact (cf. [4]) that $\beta(0, \cdot) \in L^{1}(\Omega, d v)$.

We collect the previous results to obtain a statement which is completely analogous to Theorem 3.4 (see also the Remark 3.5).

Theorem 4.10. Let $\Omega \subset \mathbb{C}^{n}$ be a BSD of genus $p$ equipped with the Bergman metric and let $f \in \operatorname{UC}(\Omega)$. Then the integral transforms $\left\{B_{\lambda}(f)\right\}_{\lambda \geq p}$ in (4.3) define a flow of real analytic functions in $\operatorname{Lip}(\Omega)$ with

$$
\lim _{\lambda \rightarrow \infty} B_{\lambda}(f)=f
$$

uniformly on $\Omega$. The Lipschitz constant of $B_{\lambda}(f)$ is dominated by $C_{\lambda}:=2 \sqrt{\lambda / p}\|f\|_{\mathrm{BMO}_{\lambda}}$. In particular, the inclusion $\operatorname{Lip}(\Omega) \subset \mathrm{UC}(\Omega)$ is dense.

Remark 4.11. In the case where $\Omega=\mathbb{B}^{n}$, it has been shown in [17] that the spaces $\mathrm{BMO}_{\lambda}^{2}\left(\mathbb{B}^{n}\right)$ in fact are independent of the weight parameter $\lambda>n$.

Remark 4.12. In our results, we have assumed that $\Omega \subset \mathbb{C}^{n}$ is an irreducible BSD. However, they remain valid for arbitrary reducible BSDs by reasonably clear modifications of the proofs for the irreducible case.

\section{Real analytic Lipschitz approximation and the Berezin measure}

Let $\Omega \subset \mathbb{C}^{n}$ be a domain equipped with a finite positive Borel measure $v$ normalized to one, i.e. $v(\Omega)=1$. We write $\mathscr{H}(\Omega)$ for the Fréchet space of all holomorphic functions on $\Omega$. Assume that the $v$-Bergman space

$$
L_{\text {an }}^{2}(\Omega, v):=\mathscr{H}(\Omega) \cap L^{2}(\Omega, v)
$$

is a Hilbert subspace of $L^{2}(\Omega, v)$ with a reproducing kernel $K: \Omega \times \Omega \rightarrow \mathbb{C}$ such that $K$ is non-vanishing on the diagonal, i.e. one has $K(z, z)>0$ for all $z \in \Omega$. We define the Berezin measure $d V_{v}$ on $\Omega$ by

$$
d V_{v}(z):=K(z, z) d v(z)
$$

Moreover, for a given bounded function $f \in L^{\infty}(\Omega, v)$ and with $z \in \Omega$ we write

$$
B_{v}(f)(z):=\frac{\langle f K(\cdot, z), K(\cdot, z)\rangle}{K(z, z)}
$$


for the Berezin transform of $f$ with respect to $L_{\text {an }}^{2}(\Omega, v)$, where $\langle\cdot, \cdot\rangle$ denotes the inner product of $L^{2}(\Omega, v)$. With the normalized reproducing kernels

$$
k_{z}(u):=\frac{K(u, z)}{\|K(\cdot, z)\|^{\frac{1}{2}}} \in L_{\mathrm{an}}^{2}(\Omega, v),
$$

where $z, u \in \Omega$, we can rewrite the Berezin transform (5.2) of $f$ as

$$
B_{v}(f)(z)=\left\langle f k_{z}, k_{z}\right\rangle=\int_{\Omega} f(u)\left|k_{z}(u)\right|^{2} d v(u) .
$$

In the next example we express these objects more explicitly in the case where $\Omega=\mathbb{C}^{n}$ or $\Omega$ is a BSD.

Example 5.1. Let $\Omega=\mathbb{C}^{n}$ be equipped with the Gaussian measure $\mu_{t}$ in formula (3.2), where $t>0$. Then we have $B_{\mu_{t}}(f)=\tilde{f}^{(t)}$ for $f \in L^{\infty}\left(\mathbb{C}^{n}\right)$ and

$$
d V_{\mu_{t}}(z)=\frac{1}{(4 \pi t)^{n}} d v(z)
$$

Consider now a BSD $\Omega \subset \mathbb{C}^{n}$ of genus $p$ which carries the standard weighted measure $d \nu_{\lambda}(z):=c_{\lambda} h(z, z)^{\lambda-p} d v(z)$ (see the notation in Section 4) with $\lambda>p-1$. Then one sees from (4.7) that $B_{v_{\lambda}}(f)=B_{\lambda}(f)$ for all $f \in L^{\infty}(\Omega)$. The Berezin measure takes the form

$$
d V_{v_{\lambda}}(z)=K_{\lambda}(z, z) d v_{\lambda}(z)=c_{\lambda} h(z, z)^{-p} d v(z) .
$$

In the last equality we have used the relation (4.2) between the weight function and the reproducing kernel of $L_{\text {an, } \lambda}^{2}(\Omega, d v)$.

Note that none of the measures (5.3) and (5.4) is finite which is a typical phenomenon in the case when $L_{\text {an }}^{2}(\Omega, v)$ is infinite dimensional. Moreover, one observes that the spaces $L^{2}\left(\mathbb{C}^{n}, V_{\mu_{t}}\right)$ and $L^{2}\left(\Omega, V_{v_{\lambda}}\right)$ are independent of the weight parameter $t$ and $\lambda$, respectively. All corresponding norms are equivalent.

As was noted earlier, the families of Berezin transforms above can be extended from bounded functions to the spaces $\operatorname{UC}\left(\mathbb{C}^{n}\right)$ (and $\operatorname{UC}(\Omega)$ ), respectively, whenever $\lambda \geq p$.

With the definition (5.1) consider now the $L^{2}$-space $L^{2}\left(\Omega, V_{v}\right)$. Then we have the following (cf. [3, Example 3.1]):

Lemma 5.2. The Berezin transform is linear and well-defined on $L^{2}\left(\Omega, V_{v}\right)$ and leaves this space invariant. More precisely, it defines a contraction on $L^{2}\left(\Omega, V_{v}\right)$.

Proof. Let $f \in L^{2}\left(\Omega, V_{v}\right)$. One can check that

$$
\int_{\Omega^{3}} \frac{1}{K(y, y)} \frac{\left|k_{u}(w)\right|^{2}}{K(u, u)} \frac{\left|k_{w}(y)\right|^{2}}{K(w, w)} d V_{v}(y) d V_{v}(w) d V_{v}(u)=v(\Omega)=1 .
$$

Hence we conclude from Tonelli's theorem that the function

$$
L(u, y):=\frac{1}{K(y, y)} \int_{\Omega}\left|k_{u}(w)\right|^{2}\left|k_{w}(y)\right|^{2} d v(w)
$$


is finite for a.e. $(u, y) \in \Omega^{2}$ with respect to the product measure $d V_{v} \otimes d V_{v}$ and non-negative. By using the identity $\left|k_{w}(u)\right|^{2}=K(u, u) K^{-1}(w, w)\left|k_{u}(w)\right|^{2}$ one finds that

$$
\int_{\Omega} L(u, y) d V_{v}(u)=\int_{\Omega} L(u, y) d V_{v}(y)=1 .
$$

Hence the two functions $F_{1}(u, y):=f(u)$ and $F_{2}(u, y):=f(y)$ are both elements of the $L^{2}$-space $L^{2}\left(\Omega \times \Omega, L(\cdot, \cdot) d V_{v} \otimes d V_{v}\right)$ with the norm

$$
\int_{\Omega^{2}}\left|F_{j}(u, y)\right|^{2} L(u, y) d V_{v} \otimes d V_{v}(u, y)=\|f\|_{L^{2}\left(\Omega, V_{v}\right)}^{2}, \quad j=1,2 .
$$

Finally, Fubini's theorem and (5.5) together with the Cauchy-Schwarz inequality shows that

$$
\begin{aligned}
\left\|B_{v}(f)\right\|_{L^{2}\left(\Omega, V_{v}\right)}^{2} & =\int_{\Omega^{3}} f(u) \overline{f(y)}\left|k_{w}(u)\right|^{2}\left|k_{w}(y)\right|^{2} d v(u) d v(y) d V_{v}(w) \\
& =\int_{\Omega^{3}} f(u) \overline{f(y)}\left|k_{u}(w)\right|^{2}\left|k_{w}(y)\right|^{2} d v(w) d V_{v}(u) d v(y) \\
& =\int_{\Omega^{2}} F_{1}(u, y) \overline{F_{2}(u, y)} L(u, y) d V_{v} \otimes d V_{v}(u, y) \\
& \leq\|f\|_{L^{2}\left(\Omega, V_{v}\right)}^{2} .
\end{aligned}
$$

This proves the lemma.

If we restrict our analysis to certain subspaces of $\mathrm{UC}\left(\mathbb{C}^{n}\right)$ and $\mathrm{UC}(\Omega)$, respectively, then we can sharpen the statements of both Theorems 3.4 and 4.10.

Theorem 5.3. Let $f \in \mathrm{UC}\left(\mathbb{C}^{n}\right) \cap L^{2}\left(\mathbb{C}^{n}, d v\right)$. Then the heat transforms $\left\{\tilde{f}^{(t)}\right\}_{t>0}$ define a flow of real analytic functions in $\operatorname{Lip}\left(\mathbb{C}^{n}\right) \cap L^{2}\left(\mathbb{C}^{n}, d v\right)$ with

$$
\lim _{t \rightarrow 0} \tilde{f}^{(t)}=f
$$

uniformly on $\mathbb{C}^{n}$. The Lipschitz constant of $\tilde{f}^{(t)}$ is dominated by

$$
D_{t}:=(4 \pi)^{-n} t^{-n-\frac{1}{2}}\|f\|_{L^{2}\left(\mathbb{C}^{n}, d v\right)} .
$$

Proof. According to Theorem 3.4, Lemma 5.2 and the first part of Example 5.1 we have

$$
\tilde{f}^{(t)} \in \operatorname{Lip}\left(\mathbb{C}^{n}\right) \cap L^{2}\left(\mathbb{C}^{n}, d v\right)
$$

for $f \in \mathrm{UC}\left(\mathbb{C}^{n}\right) \cap L^{2}\left(\mathbb{C}^{n}, d v\right)$ and all $t>0$. Let $K$ denote the reproducing kernel of the space $H^{2}\left(\mathbb{C}^{n}, d \mu\right)$. Then we obtain from $|K(u, w)|^{2} \leq K(u, u) K(w, w)$ for all $u, w \in \mathbb{C}^{n}$ that

$$
\begin{aligned}
|\operatorname{MO}(f)(u)| & \leq \widetilde{|f|^{2}}(u) \\
& =\int_{\mathbb{C}^{n}}|f(w)|^{2}\left|k_{u}(w)\right|^{2} d \mu(w) \\
& \leq \int_{\mathbb{C}^{n}}|f(w)|^{2} K(w, w) d \mu(w) \\
& =\|f\|_{L^{2}\left(\mathbb{C}^{n}, V_{\mu}\right)}^{2} .
\end{aligned}
$$


From the last estimate we conclude that $\|f\|_{\mathrm{BMO}} \leq\|f\|_{L^{2}\left(\mathbb{C}^{n}, V_{\mu}\right)}$. In particular, we have for all $t>0$,

$$
\begin{aligned}
\|f(\cdot 2 \sqrt{t})\|_{\mathrm{BMO}}^{2} & \leq\|f(\cdot 2 \sqrt{t})\|_{L^{2}\left(\mathbb{C}^{n}, V_{\mu}\right)}^{2} \\
& =\frac{1}{(4 \pi t)^{n}} \int_{\mathbb{C}^{n}}|f(2 \sqrt{t} u)|^{2} d v(u) \\
& =\frac{1}{(4 \pi t)^{2 n}}\|f\|_{L^{2}\left(\mathbb{C}^{n}, d v\right)}^{2} .
\end{aligned}
$$

A second application of Theorem 3.4 shows now that the Lipschitz constant of $\tilde{f}^{(t)}$ is dominated by $D_{t}:=(4 \pi)^{-n} t^{-n-\frac{1}{2}}\|f\|_{L^{2}\left(\mathbb{C}^{n}, d v\right)}$.

Let $\Omega \subset \mathbb{C}^{n}$ be a bounded irreducible domain of genus $p$. For any $\lambda>p-1$ consider the Berezin measure $d V_{v_{\lambda}}(z)=c_{\lambda} d \omega(z)$ on $\Omega$ in (5.4). Note that the measure

$$
d \omega(z):=h(z, z)^{-p} d v(z)
$$

is independent of the weight parameter $\lambda$. Then we have:

Theorem 5.4. Let $f \in \mathrm{UC}(\Omega) \cap L^{2}(\Omega, \omega)$. Then the integral transforms $\left\{B_{\lambda}(f)\right\}_{\lambda \geq p}$ in (4.3) define a flow of real analytic functions in $\operatorname{Lip}(\Omega) \cap L^{2}(\Omega, \omega)$ with

$$
\lim _{\lambda \rightarrow \infty} B_{\lambda}(f)=f
$$

uniformly on $\Omega$. The Lipschitz constant of $B_{\lambda}(f)$ is dominated by

$$
M_{\lambda}:=2 \sqrt{\frac{\lambda}{p \pi^{n}} \cdot \frac{\Gamma_{\Omega}(\lambda)}{\Gamma_{\Omega}\left(\lambda-\frac{n}{r}\right)}} \cdot\|f\|_{L^{2}(\Omega, \omega)},
$$

where $\Gamma_{\Omega}(\lambda)$ denotes the Gindikin Gamma function and $r$ is the rank of $\Omega$.

Proof. By the same argument as in the proof of Theorem 5.3 we conclude that

$$
\|f\|_{\mathrm{BMO}_{\lambda}} \leq \sqrt{c_{\lambda}}\|f\|_{L^{2}(\Omega, \omega)} .
$$

Hence the upper bound $M_{\lambda}$ for the Lipschitz constant of $B_{\lambda}(f)$ is obtained from Theorem 4.10 and the definition (4.1) of $c_{\lambda}$.

We close the section with some remarks on the relation between the spaces $\operatorname{BMO}^{2}(\Omega)$ and $L^{2}\left(\Omega, V_{v}\right)$ in the case where $\Omega=\mathbb{C}^{n}$ with the Gaussian measure $d \mu$ or $\Omega$ is a bounded symmetric domain of genus $p$ equipped with the measure $d v_{p}$.

In the general framework (as it was described at the beginning of this section) we denote by $P$ the orthogonal projection of $L^{2}(\Omega, v)$ onto $L_{\text {an }}^{2}(\Omega, v)$. With a (suitable) complex-valued symbol $f$ the Hankel operator $H_{f}$ is defined by

$$
H_{f}=(I-P) M_{f}: L_{\mathrm{an}}^{2}(\Omega, v) \rightarrow L_{\mathrm{an}}^{2}(\Omega, v)^{\perp} \subset L^{2}(\Omega, v),
$$

where $M_{f}$ denotes the multiplication by $f$. The following result was shown in [3, Proposition 4.1]. 
Lemma 5.5. Let $f \in L^{2}\left(\Omega, V_{v}\right)$. Then the Hankel operator $H_{f}$ is of Hilbert-Schmidt type.

Assume that $\Omega=\mathbb{C}^{n}$ or $\Omega$ is a BSD of genus $p$ equipped with the measure $d \mu$ and $d \nu_{p}$, respectively. We say that $f$ is of vanishing mean oscillation $\left(f\right.$ is in $\operatorname{VMO}^{2}(\Omega) \subset \mathrm{BMO}^{2}(\Omega)$ ) if one of the following holds:

(i) $\Omega=\mathbb{C}^{n}$ and $\lim _{z \rightarrow \infty} \operatorname{MO}(f, z)=0$,

(ii) $\Omega \subset \mathbb{C}^{n}$ a BSD and $\lim _{z \rightarrow \partial \Omega} \operatorname{MO}^{p}(f, z)=0$.

In both cases it is known that the simultaneous compactness of the Hankel operators $H_{f}$ and $H_{\bar{f}}$ implies that $f \in \mathrm{VMO}^{2}(\Omega)$ (see $\left.[1,4]\right)$. Hence Lemma 5.5 shows:

Corollary 5.6. Let $\Omega$ be a BSD. Then

$$
L^{2}(\Omega, \omega) \subset \operatorname{VMO}^{2}(\Omega) \subset \mathrm{BMO}^{2}(\Omega) .
$$

The analogous result holds for $\Omega=\mathbb{C}^{n}$.

Remark 5.7. By a direct calculation it even can be shown that for a BSD $\Omega$ of genus $p$ and $f \in L^{2}(\Omega, \omega)$ one has $\lim _{z \rightarrow \partial \Omega} \mathrm{MO}^{p}\left(|f|^{2}\right)(z)=0$. This convergence implies that $M_{f} P$ is compact on $L^{2}(\Omega, d v)$ for all $f \in L^{2}(\Omega, \omega)$. We omit the proofs here. The analogous results are true for $\Omega=\mathbb{C}^{n}$ equipped with a Gaussian measure and $f \in L^{2}\left(\mathbb{C}^{n}, d v\right)$.

\section{References}

[1] W. Bauer, Mean oscillation and Hankel operators on the Segal-Bargmann space, Integral Equations Operator Theory 52 (2005), 1-15.

[2] W. Bauer, L. A. Coburn and J. Isralowitz, Heat flow, BMO, and the compactness of Toeplitz operators, J. Funct. Anal. 259 (2010), 57-78.

[3] W. Bauer and K. Furutani, Hilbert Schmidt Hankel operators and Berezin iteration, Tokyo J. Math. 31 (2008), no. 2, 293-319.

[4] D. Békollé, C.A. Berger, L.A. Coburn and K. H. Zhu, BMO in the Bergman metric on bounded symmetric domains, J. Funct. Anal. 93 (1990), 310-350.

[5] Y. Benyamini and J. Lindenstrauss, Geometric non-linear functional analysis, Amer. Math. Soc. Colloq. Publ. 48, American Mathematical Society, Providence 2000.

[6] C.A. Berger and L.A. Coburn, Heat flow and Berezin-Toeplitz estimates, Amer. J. Math. 116 (1994), 563-590.

[7] J. Faraut, S. Kaneyuki, A. Koranyi, Q. Lu and G. Roos, Analysis and geometry on complex homogeneous domains, Progr. Math. 185, Birkhäuser, Boston 2000.

[8] J. Faraut and A. Koranyi, Function spaces and reproducing kernels on bounded symmetric domains, J. Funct. Anal. 88 (1990), no. 1, 64-89.

[9] S. Helgason, Differential geometry, Lie groups and symmetric spaces, Pure Appl. Math. 80, Academic Press, New York 1978.

[10] L. K. Hua, Harmonic analysis of functions of several complex variables in the classical domains, Transl. Math. Monogr. 6, American Mathematical Society, Providence 1963.

[11] O. Loos, Bounded symmetric domains and Jordan pairs, Math. Lectures, University of California at Irvine, Irvine 1977.

[12] M. Mitkovski, D. Suarez and B.D. Wick, The essential norm of operators on $A_{\alpha}^{p}\left(\mathbb{B}_{n}\right)$, Integral Equations Operator Theory 75 (2013), no. 2, 197-233.

[13] K. Nam, D. Zheng and C. Zhong, m-Berezin transform and compact operators, Rev. Mat. Iberoam. 22 (2006), no. 3, 867-892. 
[14] R. M. Timoney, Bloch functions in several complex variables. I, Bull. Lond. Math. Soc. 12 (1980) 241-267.

[15] H. Upmeier, Toeplitz operators and index theory in several complex variables, Oper. Theory Adv. Appl. 81, Birkhäuser, Basel 1996.

[16] K. Zhu, Analysis on Fock spaces, Grad. Texts in Math. 263, Springer, New York 2012.

[17] K. Zhu, BMO and Hankel operators on Bergman spaces, Pacific J. Math. 155 (1992), no. 2, 377-397.

Wolfram Bauer, Institut für Analysis, Leibniz Universität, Welfengarten 1, 30167 Hannover, Germany e-mail: bauer@math.uni-hannover.de

Lewis A. Coburn, Department of Mathematics, SUNY at Buffalo, New York 14260, USA

e-mail: lcoburn@buffalo.edu

Eingegangen 2. Dezember 2013 